\title{
Anabases
}

ANABASES Traditions et réceptions de l'Antiquité

$16 \mid 2012$

Varia

\section{Le péplum, et après? L’Antiquité dans les récits postmodernes}

Vivien Bessières

\section{(2) OpenEdition}

12 Journals

Édition électronique

URL : http://journals.openedition.org/anabases/3993

DOI : 10.4000/anabases.3993

ISSN : 2256-9421

Éditeur

E.R.A.S.M.E.

Édition imprimée

Date de publication : 1 octobre 2012

Pagination : 280-285

ISSN : 1774-4296

\section{Référence électronique}

Vivien Bessières, «Le péplum, et après ? L'Antiquité dans les récits postmodernes », Anabases [En ligne], 16 | 2012, mis en ligne le 01 octobre 2015, consulté le 21 octobre 2019. URL : http:// journals.openedition.org/anabases/3993 ; DOI : 10.4000/anabases.3993

Ce document a été généré automatiquement le 21 octobre 2019

(c) Anabases 


\title{
Le péplum, et après ? L'Antiquité dans les récits postmodernes
}

\author{
Vivien Bessières
}

1 Le vaste champ des recherches sur la réception de l'Antiquité dans les récits contemporains semble partagé entre deux approches.

2 La première, culturelle, historique, apparentée aux cultural studies anglo-saxonnes, s'intéresse de manière assez homogène à la réception populaire de l'Antiquité, c'est-àdire au péplum, ou epic aux États-Unis, et à toutes les formes apparentées dans d'autres genres, comme le roman, la bande dessinée ou la série télévisée historiques ${ }^{20}$. Une autre, plus spécifiquement littéraire, herméneutique, se consacre à l'étude de la réception de l'Antiquité dans des œuvres singulières, appartenant le plus souvent aux arts anciens du roman et du théâtre ou au cinéma non hollywoodien. C'est un champ hétérogène, considérant des œuvres assez différentes les unes des autres, et souvent dans des études spécifiques sur tel auteur, telle œuvre.

3 Un nouveau regard n'est-il pas possible, qui embrasse de manière synthétique ces deux points de vue? Ne peut-on analyser les œuvres de genre, gravitant autour du péplum, non plus seulement comme des objets culturels, mais aussi comme des œuvres d'art? Le plus souvent, en effet, il est question, dans ce champ de recherche, de comparer la représentation esthétique avec la vérité historique ou bien d'analyser l'idéologie plus ou moins implicite travaillant l'œuvre. Ne convient-il pas d'ajouter à cette perspective culturelle - indispensable, certes, surtout à propos de genres historiques - une perspective proprement esthétique?

4 Telles sont les questions qui ont fait l'objet de notre travail de recherche en doctorat, aboutissant à une thèse intitulée Antiquité et postmodernité - Les intertextes gréco-latins dans les arts à récit depuis les années soixante (fiction, théâtre, cinéma, série télévisée, bande dessinée) $)^{21}$. Le paradigme des cultural studies peut gagner à être confronté à un paradigme plus classique : le paradigme aristotélicien, c'est-à-dire l'idée qu'une œuvre d'art, et spécialement une œuvre de genre, ne cherche pas à représenter le vrai et ce qui est, mais le vraisemblable et ce qui doit être, au moyen de techniques et de codes appropriés $^{22}$. Les œuvres du péplum sont aussi des œuvres d'art, dans lesquelles 
l'histoire (story) compte toujours plus que l'Histoire (History), malgré leur prétention réaliste.

5 C'est à partir de cette approche générique qu'il devient possible de situer les œuvres plus singulières, qui ont un pied dans les genres populaires et un pied dans d'autres esthétiques.

6 Ainsi, des romans comme Mémoires d'Hadrien de Marguerite Yourcenar ${ }^{23}$ participent à la fois du genre du roman historique, de type péplum, et d'une vision personnelle, moderne, nourrie de genres littéraires (commentarii latins, mémoires de l'âge classique). C'est ce que nous avons appelé le "roman togé »: un roman à espace-temps antique, dans la lignée du roman historique populaire, né avec Walter Scott, mais également à style antique, imitant, pastichant des textes anciens ${ }^{24}$.

7 Des réalisateurs comme Federico Fellini ou Jean-Luc Godard proposent, eux, avec le Satyricon ${ }^{25}$ et Le Mépris $^{26}$, des œuvres très éloignées de toute esthétique générique, mais dialoguant toutes deux avec le péplum, en allant chercher le roman de Pétrone ou l' Odyssée d'Homère en guise d'anti-modèles à opposer au genre. C'est ce que nous avons appelé l'« antipéplum ».

8 C'est dans ce cadre que la notion de postmodernité trouve toute son utilité et sa pertinence. Le terme peut désigner soit une époque, commençant dans les années soixante, soit une esthétique propre à cette époque. Chez des théoriciens comme le français Jean-François Lyotard ou l'américain Fredric Jameson, deux caractéristiques définissent surtout l'esthétique postmoderne: la méfiance à l'égard des "métarécits ${ }^{27}$ ", c'est-à-dire des récits de légitimation, des mythes, des idéologies anciennes ou modernes; le recours privilégié à l'intertextualité, c'est-à-dire le jeu avec des formes dépassées, le retour plus ou moins ironique à des textes, des styles anciens, contre la recherche moderne du nouveau à tout prix ${ }^{28}$.

9 Or, beaucoup d'œuvres de notre corpus possèdent de telles caractéristiques. Avant les années soixante, que ce soit du côté du péplum ou bien du côté des arts anciens (notamment du théâtre), l'accent était plutôt mis sur le Mythe et l'Histoire dans la réception de l'Antiquité - en bref, sur des récits plutôt que sur des textes précis : un péplum ne reprenait pas un texte particulier, mais un récit historique, avec ses grands hommes ; un drame moderne, qu'il soit de Jean Cocteau, de Jean-Paul Sartre, de Thomas Stearn Eliot, d'Eugene O'Neill ${ }^{29}$, reprenait moins un texte que l'argument de la fable ${ }^{30}$ dramatique, souvent transposée dans le monde contemporain. Après les années soixante, de nombreux auteurs, de théâtre notamment, se sont mis à se méfier du Mythe et de l'Histoire ancienne, et à recourir aux textes antiques eux-mêmes, pour déconstruire les récits ${ }^{31}$. Par exemple, Les Tablettes de buis d'Apronenia Avitia de Pascal Quignard $^{32}$ racontent, sous la forme d'un journal fictif, l'histoire d'une patricienne du $\mathrm{IV}^{\mathrm{e}}$ siècle qui refuse explicitement de participer aux grands événements, à la grande Histoire - et la racontent en pastichant des styles et des textes antiques. De même, Péplum de Blutch, malgré son titre, ne représente presque rien de l'Histoire romaine, mais fonctionne comme une continuation onirique du texte du Satyricon, le citant à la lettre, ou le détournant en le collant à d'autres textes, d'autres images, de la culture populaire ou lettrée.

10 La définition d'une esthétique classique liée au genre du péplum peut s'inscrire ellemême dans un point de vue sémantique classique, essentialiste, aristotélicien : pour faire partie du genre péplum, il faut respecter un certain nombre de caractéristiques (espace-temps, types et scènes, codes du vraisemblable, idée morale). En revanche, 
l'esthétique postmoderne ne semble pas pouvoir relever d'une définition classique, mais plutôt d'une définition par "airs de famille», selon le concept de Ludwig Wittgenstein. Définir une esthétique postmoderne à travers des airs de famille revient à identifier certaines formes et enjeux qui peuvent faire partie de cette esthétique sans être obligatoires.

11 Par exemple, nous avons pu remarquer que les modernes aiment transposer la matière antique dans l'espace-temps présent, tandis que les postmodernes préfèrent en revenir à l'espace-temps antique lui-même, privilégiant en quelque sorte une esthétique de la katabasis, cette "descente aux enfers » opérée par Énée au livre IV de l'Enéide, plutôt qu'une esthétique de la nekuia, cette «invocation des morts » à soi, telle qu'Ulysse l'entreprend au chant XI de l'Odyssée. Cependant, une telle caractéristique est seulement encore un air de famille, et si elle marque une tendance, elle n'est pas non plus indispensable; on peut la trouver dans d'autres esthétiques. C'est couplée à d'autres airs de famille qu'elle finit par devenir une marque de l'esthétique postmoderne.

12 À partir de ces analyses, nous sommes parvenu à la conclusion que la matière antique dans les arts à récit avait moins perdu de son importance, depuis les années soixante, qu'elle n'avait modifié ses formes et ses enjeux. Sur un plan formel, on a même pu assister à un retour relatif aux textes antiques, et à un retour partiel à l'espace-temps antique (katabasis), au-delà des transpositions modernes (nekuia). Sur le plan des enjeux, des thèmes, l'accent a été davantage mis sur la représentation d'identités individuelles et sociales, telles notamment l'identité de genre et l'identité de sexualité, ces thèmes étant étroitement liés aux questions récurrentes de la théorie postmoderne autour du genre féminin et de l'homosexualité.

13 Le genre du péplum et la modernité du premier $\mathrm{xx}^{\mathrm{e}}$ siècle s'intéressaient surtout aux aspects moraux et politiques dans leur traitement de l'Antiquité, posant la question du pouvoir et de la liberté. Les œuvres postmodernes considèrent plutôt l'Antiquité à un niveau historique et social, soit comme une dystopie, un espace-temps originaire des dominations identitaires, qu'elles soient de genre, de sexualité, de culture en général, soit comme une utopie, un espace-temps alternatif, proposant d'autres modèles identitaires, d'autres façons de faire avec le genre féminin, l'homosexualité, l'autre en général.

Cependant, à côté d'œuvres qui semblent exemplaires d'une nouvelle esthétique, d'une nouvelle façon de voir et de faire avec l'Antiquité, il en est d'autres qui, tout en appartenant à l'époque postmoderne, ne relèvent pas de l'esthétique postmoderne : des œuvres de genre, avec des codes, des types, des scènes attendus; ou bien des œuvres plus singulières, mais qui ne se méfient pas du mythe, au contraire, et recourent peu aux textes antiques tels quels, participant d'une esthétique similaire au modernisme du premier $\mathrm{xx}^{\mathrm{e}}$ siècle, avec ses adaptations de tragédies, par exemple.

15 Il faut ainsi distinguer entre œuvres postmodernes au sens large, c'est-à-dire d'époque postmoderne, et œuvres postmodernes au sens strict, d'esthétique postmoderne. Et l'époque postmoderne apparaît alors comme ce temps éclectique où différentes esthétiques peuvent être mises sur le même plan sans que l'une ait a priori plus de valeur que l'autre.

Dans Le Vol de l'histoire - Comment l'Europe a imposé le récit de son passé au reste du monde ${ }^{33}$, Jack Goody formule la thèse que certaines périodes comme l'Antiquité, la Renaissance, certaines valeurs comme l'Humanisme, la Démocratie, ont été confisquées par 
l'Occident à d'autres civilisations telles que le Proche-Orient, l'Inde, la Chine, qui les ont pourtant aussi connues. À tous ces mots, et d'autres, l'Occident a mis des majuscules, comme s'il n'y avait qu'une seule Antiquité, qu'une seule Renaissance, les nôtres, l'Antiquité gréco-latine, la Renaissance européenne, comme si nous avions inventé les idées d'humanisme et de démocratie, qui seraient nées en Grèce, transmises par Rome à l'Europe et l'Amérique occidentales - en passant très vite par l'Islam, dont on essayera éventuellement de minimiser l'apport... C'est dans un même esprit démocratique que nous avons souhaité faire dialoguer entre eux arts anciens et arts nouveaux ${ }^{34}$, lettres classiques et lettres contemporaines. La réduction des antiquités à l'Antiquité grécolatine s'est en effet parfois doublée d'une réduction interne de l'Antiquité gréco-latine aux seules lettres classiques, à la seule République des Lettres ${ }^{35}$. Dans cette République, on se parle par textes interposés et l'on se prend souvent à pester contre tous ces médias qui font que les jeunes ne lisent plus. Les images sont mal perçues, surtout à la télévision, tandis que les textes sont valorisés, surtout les "grands textes». Cette réduction humaniste a des racines profondes, dans l'Antiquité même. Que l'on soit pour ou contre les médias de masse, que l'on se range du côté du "oui » progressiste postmoderne ou du "non " réactionnaire moderne, on aura tendance à opposer deux sortes de cultures, littéraires et spectaculaires, textuelles et orales, élitaires et populaires, à l'instar de ces écrivains romains civilisés qui s'envoient des lettres pardessus le tumulte barbare du cirque. Dans ses Règles pour le parc humain, le philosophe postmoderne Peter Sloterdijk continue de renvoyer dos à dos ce qui serait une culture de la «domestication" par les lettres et une culture de la «bestialisation" par le spectacle $^{36}$.

17 Cependant, depuis les années soixante, beaucoup d'études dans le domaine de l'Antiquité, consacrées par exemple à l'épopée comme tradition orale, au théâtre comme performance spectaculaire, nous ont montré combien cette dichotomie ne résistait pas à l'examen. Florence Dupont, notamment, a pu mettre en valeur tout ce que la tragédie grecque ne doit pas au texte ${ }^{37}$, ou tout ce que la tragédie romaine doit au spectacle des jeux, des ludi, dont elle fait partie, en tant que «jeu scénique », au même titre que les jeux du cirque ${ }^{38}$. Le théâtre antique, partiellement confisqué par l'idéologie de l'Antiquité comme texte, c'était avant tout du spectacle - à ranger donc dans la catégorie de la bestialité ?

18 Avec l'époque postmoderne, de même que l'Antiquité gréco-latine s'est trouvée décentrée à côté d'autres antiquités, les lettres classiques se sont trouvées décentrées à côté de tout ce qui n'était pas des « lettres » et des « classiques » : il n'y avait pas d'un côté les Grecs et de l'autre les barbares, mais un échange continuel, une interpénétration de toutes les antiquités; il n'y avait pas d'un côté les "Lettres classiques » et de l'autre du divertissement bestial, des jeux du cirque, mais toute une culture où l'un n'existe pas sans l'autre - et la tradition orale, populaire, spectaculaire, n'a pas seulement mené sa vie de bête à côté d'une tradition lettrée qui serait seule civilisée, mais beaucoup de grands noms qui ont leur place de choix dans la bibliothèque humaniste appartiennent en fait aussi à cette supposée sous-culture de masse.

19 Aussi, plutôt que d'une République des Lettres, peut-être faudrait-il envisager une démocratie des arts, pour l'Antiquité comme pour la postmodernité ? C'est dans le sillage d'une telle mise à plat qu'a souhaité s'inscrire notre thèse, invitant à réviser ses jugements a priori sur la valeur de telle ou telle œuvre pour la seule raison qu'elle serait 
ancienne ou moderne, littéraire ou visuelle, élitaire ou populaire, singulière ou générique.

\section{NOTES}

20. Les références sont nombreuses. Citons, par exemple, pour le domaine francophone : L. AKNIN, LE PÉPLUM, PARIS, ARMAND COLIN, 2009 ; C. AZIZA, GUIDE DE L'ANTIQUITÉ IMAGINAIRE: ROMAN, CINÉMA, BANDE DESSINÉE, PARIS, LES BELLES LETTRES, 2008; H. DUMONT, L'ANTIQUITÉ AU CINÉMA : VÉRITÉS, LÉGENDES ET MANIPULATIONS, PARIS, NOUVEAU MONDE, 2009 ; AINSI QUE, POUR LE DOMAINE ANGLOPHONE : M. WINKLER ÉD., CLASSICAL MYTH \& CULTURE IN THE CINEMA, OXFORD, OXFORD UNIVERSITY PRESS, 2001 ; M. WYKE, PROJECTING THE PAST : ANCIENT ROME, CINEMA, AND HISTORY, NEW YORK, ROUTLEDGE, 1997.

21. Thèse de Doctorat de Lettres, préparée sous la direction de $M$. le Professeur Jacques Dürrenmatt, soutenue le 30 septembre 2011 à l'Université Toulouse 2-Le Mirail.

22. Voir Aristote, Poétique, 1451b.

23. M. YOURCENAR, MÉMOIRES D'HADRIEN, PARIS, PLON, 1958.

24. Pour le roman historique, voir notamment l'ouvrage ancien mais fondamental de Lukács : G. LUKÁCS, DER HISTORISCHE ROMAN, BERLIN, AUFBAU VERLAG, 1956; LE ROMAN HISTORIQUE, TRAD. ROBERT SAILLEY, PARIS, PAYOT, 1965. POUR UNE DÉFINITION DU STYLE DES MÉMOIRES D’HADRIEN COMME ORATIO TOGATA, «STYLE TOGÉ », VOIR YOURCENAR ELLE-MÊME : M. YOURCENAR, «TON ET LANGAGE DANS LE ROMAN HISTORIQUE », NRF, 238, OCT. 1972, P. 101-123, REPRIS DANS LE TEMPS CE GRAND SCULPTEUR, PARIS, GALLIMARD, 1972, P. 32-38.

25. F. FELLINI, FELLINI SATYRICON, IT/FR, P.E.A. (ALBERTO GRIMALDI) - ARTISTES ASSOCIÉS, 1969.

26. J.-L. GODARD, LE MÉPRIS / IL DISPREZZO, FR/IT, GEORGES DE BEAUREGARD - JOSEPH E. LEVINE - CARLO PONTI ROME-PARIS FILMS - LES FILMS CONCORDIA - COMPAGNIA CINEMATOGRAFICA CHAMPION, 1963.

27. Voir J.-F. LYOTARD, LA CONDITION POSTMODERNE : RAPPORT SUR LE SAVOIR, PARIS, MINUIT, « CRITIQUE », 1978 ; LE POSTMODERNE EXPLIQUÉ AUX ENFANTS : CORRESPONDANCE 1982-1985, PARIS, GALILÉE, 1988.

28. Voir F. JAMESON, «POSTMODERNISM AND CONSUMER SOCIETY» (1984-1988), P. 1-20, THE CULTURAL TURN: SELECTED WRITINGS ON THE POSTMODERN 1983-1998, LONDON, VERSO, 1998.

29. Voir J. COCTEAU, LA MACHINE INFERNALE, DIR. LOUIS JOUVET, PARIS, COMÉDIE DES CHAMPS-ÉLYSÉES, 1934 ; J.-P. SARTRE, LES MOUCHES, DIR. CHARLES DULLIN, PARIS, THÉÂTRE DE LA CITÉ, 1943 ; T. S. ELIOT, THE COCKTAIL PARTY, DIR. E. MARTIN BROWNE, EDINBURGH, EDINBURGH FESTIVAL, 1949; E. O'NEILL, MOURNING BECOMES ELECTRA, DIR. PHILIP MOELLER, NEW YORK, GUILD THEATRE, BROADWAY, 1931.

30. La fable est, dans le vocabulaire théâtral, l'histoire que raconte la pièce, traduction du mythos de la Poétique: Aristote, Poétique, 1450a. Voir A. UBERSFELD, LES TERMES CLÉS DE L'ANALYSE DU THÉÂTRE, PARIS, SEUIL, 1996, P. 41-42.

31. Sur l'actualité des tragédies antiques dans le théâtre contemporain, voir P. VASSEURLEGANGNEUX, LES TRAGÉDIES GRECQUES SUR LA SCÈNE MODERNE: UNE UTOPIE THÉÂTRALE, VILLENEUVE D'ASCQ, PRESSES UNIVERSITAIRES DU SEPTENTRION, 2004 ; E. HALL, F. MACINTOSH ET A. WRIGLEY ÉD., DIONYSUS SINCE 69 : GREEK TRAGEDY AT THE DAWN OF THE THIRD MILLENNIUM, OXFORD, OXFORD UNIVERSITY PRESS, 2004.

32. P. QUIGNARD, LES TABLETTES DE BUIS D'APRONENIA AVITIA, PARIS, GALLIMARD, 1984.

33. J. GOODY, THE THEFT OF HISTORY, CAMBRIDGE, CAMBRIDGE UNIVERSITY PRESS, 2006; LE VOL DE L'HISTOIRE COMMENT L'EUROPE A IMPOSÉ LE RÉCIT DE SON PASSÉ AU RESTE DU MONDE, PARIS, GALLIMARD, « ESSAIS », 2010. 
34. Pour la distinction entre arts anciens et arts nouveaux, voir T. GARCIA, ARTS ANCIENS, ARTS NOUVEAUX. LES FORMES DE NOS REPRÉSENTATIONS DE L'INVENTION DE LA PHOTOGRAPHIE À AUJOURD'HUI, 2 TOMES, THÈSE DE PHILOSOPHIE, UNIVERSITÉ DE PICARDIE, 2008; T. GARCIA, FORME ET OBJET. UN TRAITÉ DES CHOSES, PARIS, PUF, « MÉTAPHYSIQUES », 2011, P. 283-304.

35. Sur la notion de République des Lettres, voir, entre autres, P. CASANOVA, LA RÉPUBLIQUE MONDIALE DES LETTRES, PARIS, SEUIL, 1999; M. FUMARoli, «LA RÉPUBLIQUE DES LETTRES », P. 131-150, DIOGÈNE, № 143, JUILLETSEPTEMBRE 1988.

36. P. SLOTERDIJK, REGELN FÜR DEN MENSCHENPARK. EIN ANTWORTSCHREIBEN ZU HEIDEGGERS BRIEF ÜBER DEN HUMANISMUS, FRANKFURT, SUHRKAMP, 1999 ; RÈGLES POUR LE PARC HUMAIN. UNE LETTRE EN RÉPONSE À LA LETTRE SUR L'HUMANISME DE HEIDEGGER, TRAD. OLIVIER MANNONI, PARIS, MILLE ET UNE NUITS, « LA PETITE COLLECTION », 2000.

37. Voir F. DUPONT, L'INSIGNIFIANCE TRAGIQUE, PARIS, LE PROMENEUR, 2001.

38. Voir F. DUPONT, LES MONSTRES DE SÉNĖQUE, PARIS, BELIN, « L'ANTIQUITÉ AU PRÉSENT », 1995.

\section{AUTEUR}

\section{VIVIEN BESSIÈRES}

Docteur en Lettres modernes, ATER à l'Université Toulouse 2 - Le Mirail vivienbessieres@yahoo.fr 